\title{
Microwave emission from the trapped and precipitated electrons in solar bursts ${ }^{\star}$
}

\author{
J. E. R. Costa ${ }^{1,2, \star \star}$ and A. C. Rosal ${ }^{1,2}$ \\ ${ }^{1}$ INPE - Instituto Nacional de Pesquisas Espaciais, Brazil \\ e-mail: jercosta@craam.mackenzie.br \\ 2 IPM/CRAAM - Instituto Presbiteriano Mackenzie/Centro de Rádio Astronomia e Astrofísica Mackenzie, Brazil
}

Received 28 October 2004 / Accepted 7 February 2005

\begin{abstract}
We analyzed the microwave spectra of a sample of 13 solar flares out of 40 events observed by the Nobeyama Radio Polarimeter in the period of August 8, 1998 through November 24, 2001. The solar burst time profiles were filtered to separate the slow component from the fast structures which were associated with the emission from trapped particles and to the emission from the minor precipitating population, respectively. The spectra of both components suggest a trap in a region with a mean magnetic field of about $600 \mathrm{G}$ and a minor emission from the loop legs with a magnetic field of $1100 \mathrm{G}$, resulting in a mirror ratio of about two. The spectral indices of the optically thin emission from these two regions indicate pitch angle diffusion by Coulomb collisions.
\end{abstract}

Key words. Sun: flares - Sun: activity - Sun: magnetic fields - Sun: particle emission - Sun: radio radiation

\section{Introduction}

Non-thermal electrons are accelerated during solar flares as a consequence of an impulsive energy release. The impulsive phase signatures of solar flares in the microwave and the hard $\mathrm{X}$-rays bands have been intensively analyzed and support the hypothesis of a common origin based on the emitting electron number and emission time profiles (e.g., Kundu 1961; Gary 1985).

Downward/upward electron streams have early been suggested to explain hard X-rays (e.g., Brown 1973; Datlowe \& Lin 1973). Although historically the up or downstream of electrons with continuous or instantaneous injection in a single or multiple steps were first considered in the flare modeling of hard X-rays, the trap plus precipitation model seemed to gain more attention due to its ability to explain a smoother microwave emission time profile. Moreover, the precipitating electrons from the trap incorporate the generally more accepted thick target model for hard X-ray emission (Melrose \& Brown 1976). Thus, the solar flare models seems to favor a trap to explain the microwave emission as opposed to continuous injections (e.g., Takakura \& Kai 1966) and the trap plus precipitation for hard X-rays (e.g., Melrose \& Brown 1976), with emphasis on the precipitation. However, the uncertainties in the diagnostics still do not allow us to derive any final strong conclusion about this matter.

* The complete Table 1 is only available in electronic form at http: //www. edpsciences.org

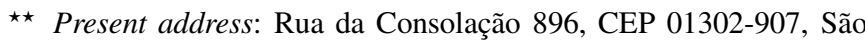
Paulo, Brazil.
The trapping time from the collisional particle lifetime can be easily inferred from most of the kinetic equation analysis. In the levels of the solar atmosphere where the hard $\mathrm{X}$-ray/microwave emission originates, the ambient density is in general higher than $10^{10}$ electrons $/ \mathrm{cm}^{3}$ which results in a collisional deflection time greater than $5 \mathrm{~s}$ for electron energies higher than $100 \mathrm{keV}$ (e.g., Lin \& Johns 1993). For more typical ambient density in the flaring region, for example $10^{11}$ electrons $/ \mathrm{cm}^{3}$, the collisional deflection time can be tens of seconds for the range of energy $(\epsilon)$ relevant for microwave emission $(\epsilon \geq 100 \mathrm{keV})$.

The light curves of hard X-ray and microwave emission present temporal features with time scales of about one second or less on top of a gradual bulk emission (Kiplinger et al. 1983; Kaufmann 1978; Correia \& Kaufmann 1987). In the paper of Lin \& Johns (1993), the importance of the inferred impulsive component above $40 \mathrm{keV}$ electrons was $1 / 6$ ( $17 \%$ ) of the gradual component. From the microwave observations, the fraction of flux in fast $(\leq 1 \mathrm{~s})$ structures in the light curve was measured in the range of $1-50 \%$ for frequencies from $5-90 \mathrm{GHz}$, respectively (Correia \& Kaufmann 1987). In hard X-rays similar pulsed emission with pulses of the order of $50 \%$ of the flux intensity and durations of 0.3 to $3 \mathrm{~s}$ has been measured and analyzed by Aschwanden et al. (1995). Thus, the main time scales for these fast structures are seconds or less over bursts with durations longer than tens of seconds and their relative importances are of the order of $50 \%$ or less of the total emission.

On the other hand, the good correlation among the different energy bands in X-rays has early presented time delays 
between the different photon energies (Hoyng et al. 1976; Bai \& Ramaty 1979). Also, hard X-ray emission showed time differences in comparison with the microwave emission (Kaufmann et al. 1982, 1983) that included time differences among different frequencies in microwaves. Based on the separation of the fast and gradual components of the hard X-ray emission, Aschwanden (1998) has associated the time delays in the fast structures of hard X-rays with the particle time of flight to infer the composition of the trapped and precipitated particles in solar flares.

Many authors have worked on the electron kinetic equation to infer the particle number distribution during solar flares using hard X-rays and/or microwaves in a trap plus precipitation model (Melrose \& Brown 1976; MacKinnon et al. 1983; Leach \& Petrosian 1981, 1983; Lu \& Petrosian 1988; Lee \& Gary 2000). The main weakness of such an approach resides in the need to make assumptions for the unknowns such as: source function time profile and duration of the injected electrons, beaming (pitch angle distribution) and the plasma ambient spatial structure such as density and magnetic field. However, using typical time scales, scale heights and homogeneous pitch angle distribution some calculation can be made.

Here we propose an analysis based on the $B^{2}$ dependence of gyro-synchrotron emission of the microwave spectra and the separability of the emission of the fast component from the bulk emission of the gradually increasing number of trapped electrons. The spatial difference of the magnetic induction from the top to the foot-point of the field line structure must result in different microwave spectra that can be analyzed to infer the local magnetic field and the non-thermal electrons properties. We used solar flare observations in seven microwave frequencies from the Nobeyama Radio Observatory (NRO) in the period of March, 1998 to December, 2000, resulting in a sample of about 100 flares.

In Sect. 2 we present the data analysis and in Sect. 3 the filter of the slow and fast time components is discussed. The magnetic field is discussed in Sect. 4, whereas the viewing angle of the magnetic loop is presented in Sect. 5. Finally, the spectral analysis and the results are presented in Sects. 6 and 7, respectively.

\section{Data analysis}

The Radio Polarimeter of the NRO (NORP) observed 123 events in the period of March, 1998 to December, 2000, in both circular polarization modes. Roughly 30 events presented problems such as missing frequencies, had data interruption or were too noisy to be analyzed. The sensitivity of the NORP radiometers is about $10 \mathrm{sfu}$. $\left(1 \mathrm{sfu}=10^{-22} \mathrm{~W} \mathrm{~m}^{-2} \mathrm{~Hz}^{-1}\right)$ for the higher frequencies (Nakajima et al. 1985) and better for the lower ones. For the separation of the fast and slow components, we typically needed events with flux densities higher than $30 \mathrm{sfu}$ for the majority of the observed frequencies due to the relative weakness of the fast component, which is $\leq 50 \%$ of the instantaneous flux density (e.g., Correia \& Kaufmann 1987). This flux density limitation and some further considerations on the spectral fit decreased our sample to 40 possible events.
Our expectation for the two components is to reveal two different scenarios from the inferred source parameters. The association of the fast component with a different region is a consequence of analyzing the electrons escaping from the trap and thereby reaching regions outside the trapped volume. Time differences in the emission signatures reflect mostly the time scale of the time of flight and/or diffusion time (precipitation or particle loss). Precipitating particles are those with pitch angles in the loss cone. The loss cone angle is the minimum pitch angle an electron has to have in order to be reflected back to the trapping volume (Melrose \& Brown 1976), i.e., with lower pitch angle the electron reaches the higher density levels were it loses most of its energy collisionally. Electrons with energy in the range of relevance for microwave emission $(\approx 100 \mathrm{keV}$ or higher) in the loss cone (any reasonable loss cone below $50^{\circ}$ ) would have a time of flight (time to travel throughout the loop length) of about one second or less (e.g., Aschwanden 1998). Thus, independent of their energy, the precipitating electrons produce microwave emission with fast time scales in comparison to trap times that are of the order of tens of seconds or more. The same idea may be applied even for the electrons that do not bypass the trapping with direct flight from the acceleration site to the foot-point, but reflect backwards to the trapping region and have their pitch angle diffused into the loss cone in showers on a time scale shorter than the full trapping time. Studying hard X-ray pulses in the range of $0.3-3 \mathrm{~s}$, Aschwanden et al. (1995) showed that the pulse durations were independent of their energy. This favored causes due to acceleration properties of the pulse instead of trapping properties. Trap times are another typical unknown, but collisional pitch angle diffusion has been explored in trap plus precipitation models (e.g., Lin \& Johns 1993; Aschwanden 1998). Collisional pitch angle diffusion occurs at a rate of twice the rate of collisional energy loss (Trubnikov 1965). Thus, the trap time is of the order of tens of seconds for typical ambient densities $\left(\approx 10^{11}\right.$ electrons $\left.\mathrm{cm}^{-3}\right)$ and energy of microwave-emitting electrons ( $\approx 100 \mathrm{keV}$ or higher). If one has to use a trap to model the microwave emission, the trap time from single or multiple injections should be able to explain the flare duration itself. This duration is typically much longer than the fast structure time scales. This difference in the time scales of both components is important to allow their separation.

In order to use a filter to separate both components of the observed total flux density, we had to assume from our discussion above that the precipitating electrons do not contribute much to the total emission during the upper atmospheric part of the electron flight. Due to the strong dependence of the gyrosynchrotron emission on the magnetic field this should be a good first order approximation.

Flare images at 17 and $34 \mathrm{GHz}$ from the Nobeyama Radioheliograph are also used in Sect. 5 to determine the loop footpoint orientation. While these images clearly distinguish between the footpoint and looptop emissions spatially, they cannot be used to construct spectra, which can only be done with the spatially integrated flux densities (the light curves) at the seven frequencies measured by the Nobeyama Radio Polarimeters. 
We note that the optically thick emission from the higher atmosphere (looptop) would cover most of the emission from below. However, most of our analysis here is based on the spectrum peak frequency, at which neither looptop nor footpoints should have a large optical depth. Thus, only a qualitative analysis of the spectral index of the optically thick part of the spectrum can be done. However, the condition is less restrictive because looking from the top the lower magnetic fields (looptop) would result in a transition frequency from optically thick to thin emission lower than the transition for higher magnetic fields (footpoints), and this will not prevent us from seeing optically thick emission from the footpoints.

\section{Filter}

The larger the differences in time scale of the components, the easier the separation for a digital filter. However, as discussed in Costa et al. (1990), due to the combination of the emissions from the trap and the precipitation regions, the running mean is a better representation of the slow component in order to preserve the original peak times. The problem with the standard running mean is that the filtered (smoothed) component is at the mean of the original signal, thus implying negative values for the fast component. To remove this inconsistency we applied a modification suggested by Raulin et al. (1991). There, the running mean intensity is computed over a specified time interval $(\tau)$ and for each interval the minimum between the original signal and the mean value is taken. This procedure is iterative and re-scales the running mean result, searching for the minimum in other time scales larger than the specified interval $(\tau)$ up to $5 \tau$. The method preserves the characteristics of the running mean filter and scales the smoothed component to be lower than the majority of the local minima of the structured original light curve. The fast component is then obtained by subtracting the gradual component from the original signal. We applied this modified running mean $(M R M)$ in a simple numerical test as shown in the example in Fig. 1. The cutoff period $(\tau)$ is the number of elements in the modified running mean which were used to separate both components. In this figure we show three cutoffs in panels $b, c$ and d where the mean error (differences divided by the maximum amplitude in percents) are $(15,-21 \%),(1,-2 \%)$ and $(-7,10 \%)$ for (gradual, fast) components, respectively. The negative/positive values are for underestimation/overestimation, respectively. In Fig. 1d we clearly see the gradual component also being smoothed. The main concern in the separation of the two components is to identify the cutoff time scale $(\tau)$ that recovers the original amplitude of both components. In the test shown the highly structured component has only periods shorter than 30 samples and the gradual component has a half maximum duration of 200 samples. Thus, the optimum cutoff period should be found in $30 \leq \tau \leq 200$ samples. We can see from our example in Fig. 1c a reasonable separation at $\tau \approx 30$ samples, whereas for $\tau=5$ and 40 the fast amplitude degrades to wrong values. In our burst sample we expect a more complex composition of the fast and slow components. This is mainly because the time scales of the gradual and fast components may not be very different and also because the scales are certainly dependent on the electron energy,
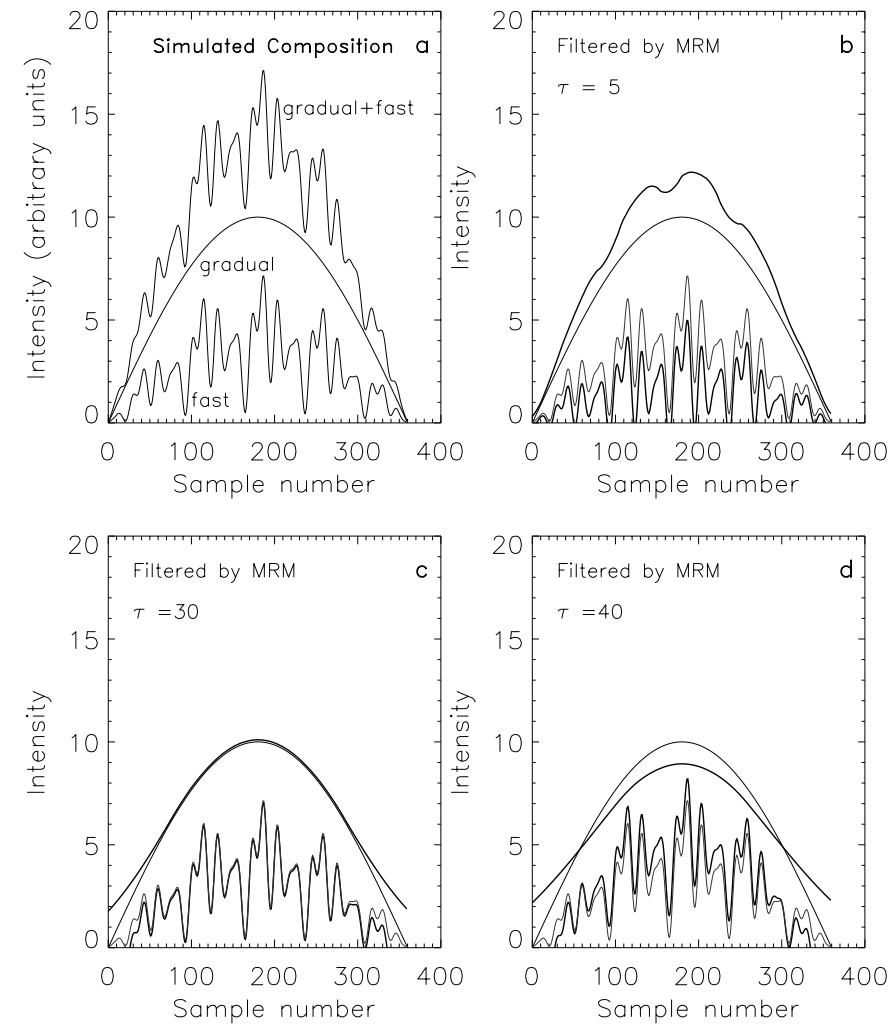

Fig. 1. Numerical test of the $M R M$ filter. In a) is the composition of a gradual component with half maximum duration of 200 samples and the fast component with mean pulse duration less than 30 samples. In the plots b), c) and d) (thick lines) are the separated components (gradual and fast) using the filter $M R M$ with $\tau$ samples in the running mean. In b), c) and d) the thin lines are the original components as shown in a), for comparison.

resulting in time delays. Energy-dependent (or frequencydependent) time delays may be a property of the accelerator or due to the time of flight of the particles. To partially overcome possible difficulties with the time delays of frequencies being different we took the sum of the maximum cross-correlation coefficients of a reference channel with the other channels, independent of the time lag. Varying the cutoff period $\tau$ from $0.2 \mathrm{~s}$ to $4 \mathrm{~s}$ we searched for the period where the sum of the maximum cross-correlation coefficients $\left(\mathrm{C}_{\mathrm{T}}\right)$ ceases to increase. Roughly, this occurs when the growth rate decreases to one third of its maximum. We do not expect that this solution is unique; however, the cutoff $\tau$ was always limited to the discussed range and we will look for tendencies in our results that are statistically well defined. In our sample of events we found 40 solar flares with amplitude of both components strong enough to construct the gyro-synchrotron spectra. Some of the bursts with very short duration had $\tau$ out of this range, as shown in Table 1. The name of the bursts contains the year (y), month $(\mathrm{m})$, day $(\mathrm{d})$, hour $(\mathrm{h})$, minute $(\mathrm{m})$ of the event with the format: norpyyyymmdd_hhmm. As an example, we plotted in Fig. 2 the burst of April 8, 2000, at 02:38 UT with the gradual and fast components separated by the MRM filter with $\tau=2.9 \mathrm{~s}$, as given in Table 1 . We can see in the $17 \mathrm{GHz}$ emission (spectrum peak) two main structures in the fast component 

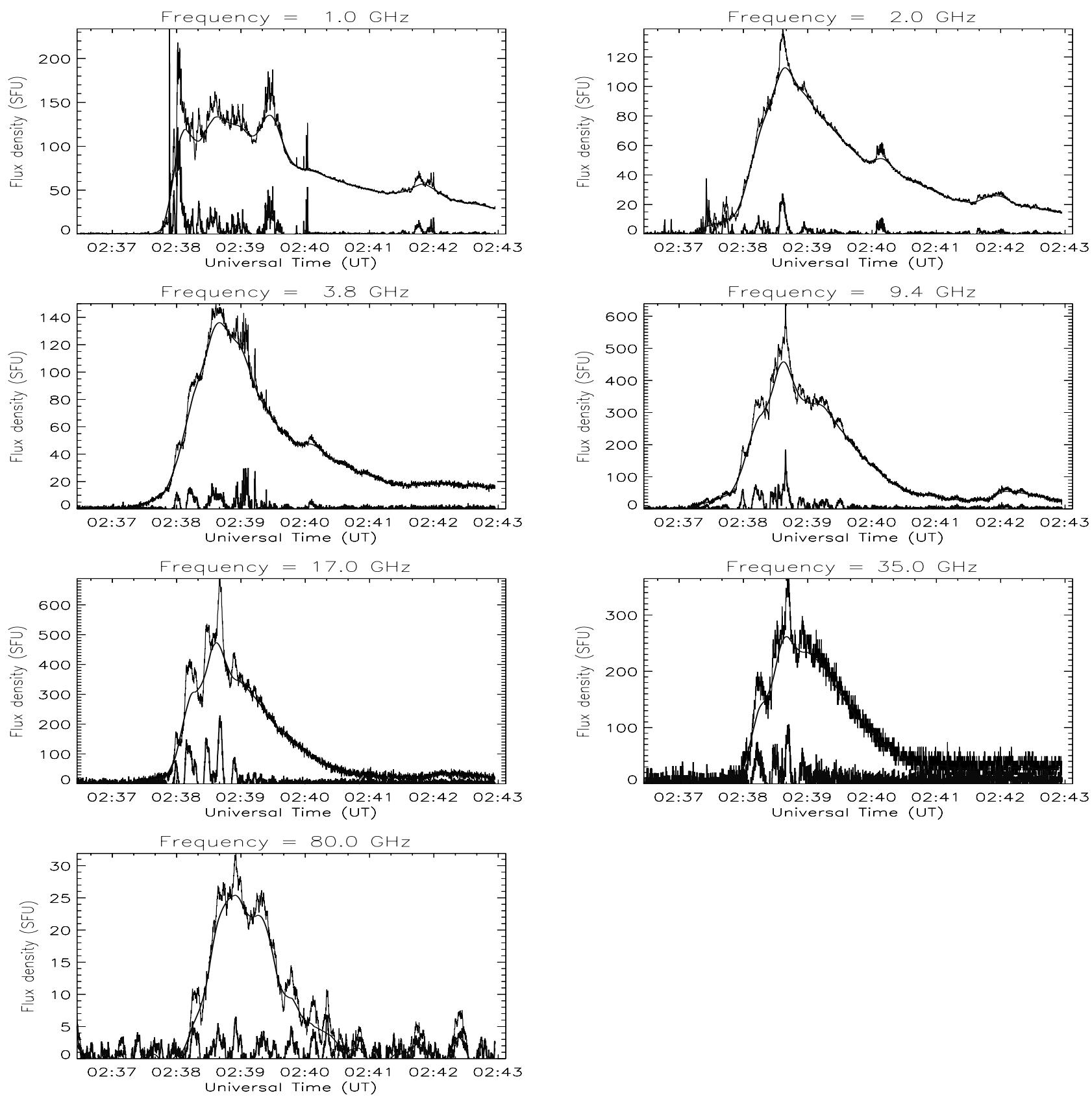

Fig. 2. The solar burst of April 8, 2000. In seven radio frequencies observed by the Nobeyama Radio Observatory. The plot lines show the gradual and fast components obtained by the $M R M$ filter with $\tau=2.9 \mathrm{~s}$ overplotted on the total observed flux density.

that is also present in all frequencies above $1 \mathrm{GHz}$. The spectra of these fast and gradual components are shown in Fig. 3 for the two main peaks of the fast components during the rise and peak times of this burst.

\section{Inferred magnetic fields}

The magnetic induction present in the flare region can be inferred from the spectrum peak (e.g., Dulk \& Marsh 1982). For a homogeneous magnetic field, the spectrum peak is well defined and may be used for the inference of the field induction whenever we know the other source parameters that are also important for the peak frequency (see discussion in Stäli et al. 1989). However, the peak frequency depends more strongly on the magnetic induction and has been widely used to make magnetic field inferences.

A non-homogeneous magnetic field may broaden the spectrum mainly due to changes in the optically thick spectral index (Klein et al. 1986). Thus, for non-homogeneous magnetic fields the inferred induction may be interpreted as an effective value when the observation is fit, for example, by the four parameter function given by Stäli et al. (1989). Simple formulas are given for the gyro-synchrotron spectral peak in Dulk \& Marsh (1982) where the peak is derived for an opacity equal to one. This simplification causes the peak to be uncertain mostly for the cases where the optically thin spectral index is low. Also, Dulk's equations are not applicable for a spectral peak 
Table 1. Analyzed events. The name used is taken from the flare list on the NRO web-site: solar.nro.nao.ac.jp/norp/html/event/. The second column has the cutoff period in seconds and the third, fourth columns are the flare duration and the spectrum peak flux density, respectively.

\begin{tabular}{ccccc}
\hline \hline$\#$ & Event & $\begin{array}{c}\tau \\
(\mathrm{s})\end{array}$ & $\begin{array}{c}\text { Duration } \\
(\mathrm{s})\end{array}$ & $\begin{array}{c}\text { Intensity } \\
(\mathrm{sfu})\end{array}$ \\
\hline 7 & norp19990216_0001 & 1.9 & 241 & 245 \\
12 & norp19990702_0133 & 3.4 & 332 & 197 \\
13 & norp19990728_0156 & 3.5 & 933 & 305 \\
16 & norp19990825_0135 & 0.7 & 55 & 132 \\
17 & norp19990828_0056 & 1.3 & 593 & 1195 \\
27 & norp20000313_0503 & 1.5 & 65 & 26 \\
30 & norp20000408_0238 & 2.9 & 313 & 688 \\
32 & norp20000623_0404 & 1.5 & 293 & 536 \\
33 & norp20000721_0522 & 1.2 & 67 & 36 \\
34 & norp20000725_0247 & 1.4 & 195 & 3019 \\
36 & norp20000916_0415 & 2.8 & 1022 & 2778 \\
39 & norp20001123_2321 & 2.4 & 154 & 697 \\
40 & norp20001124_0459 & 2.3 & 135 & 8638 \\
\hline
\end{tabular}
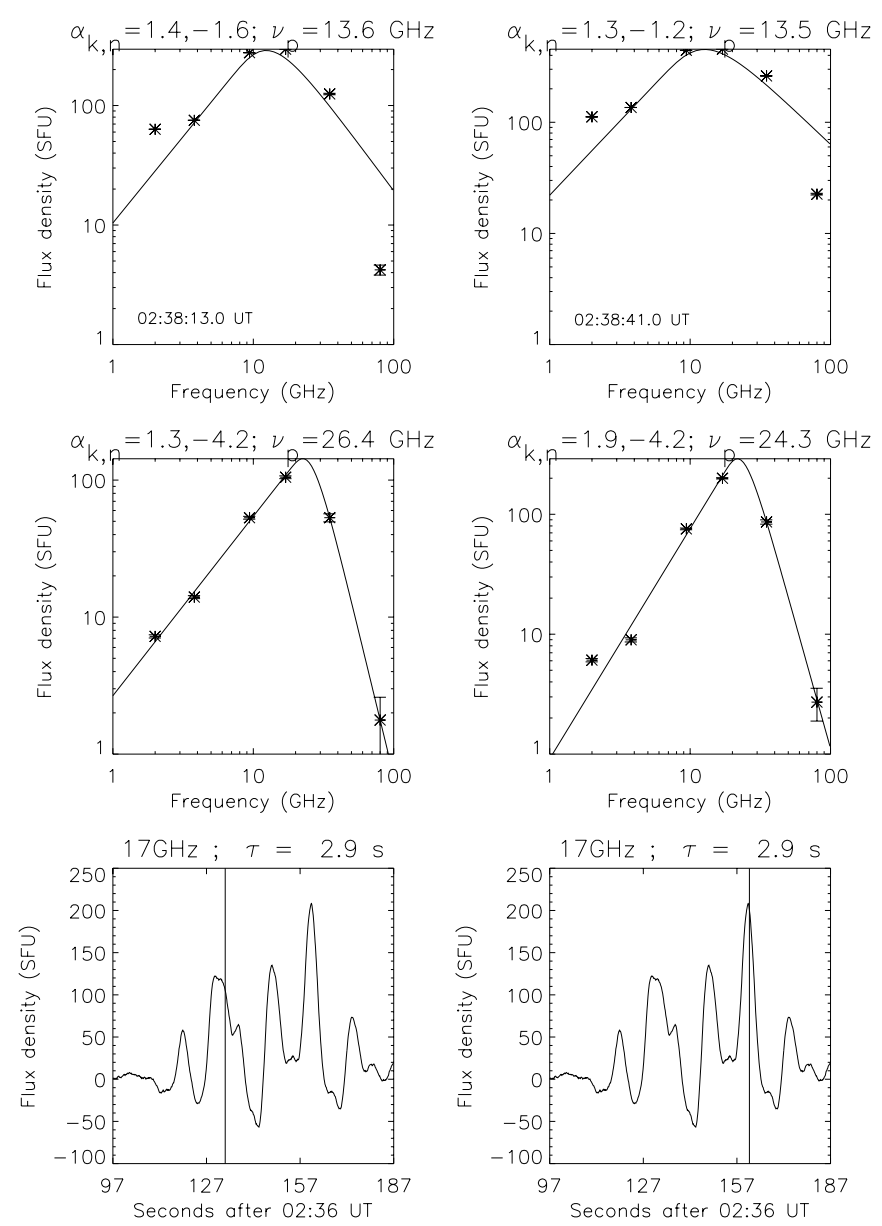

Fig. 3. Spectra of the gradual (top two graphics) and fast (two graphics in the middle) components at the peak of the two main fast structures on flare of 08 of April of 2000. On top of each spectrum is given the spectral indices of optically thick regime $\left(\alpha_{\mathrm{k}}\right)$ and optically thin $\left(\alpha_{\mathrm{n}}\right)$ and the spectrum peak frequency $\left(v_{\mathrm{p}}\right)$. The two bottom plots are the flux density of the fast component at $17 \mathrm{GHz}$ and the vertical bars indicate the times the spectra were taken. occurring below the tenth harmonic number of the electron gyro-frequency which excludes most of flares in our analysis here. For example, for magnetic fields of the order of 500 Gauss the tenth harmonic of the gyro-frequency is higher than $14 \mathrm{GHz}$, i.e., higher than the typical spectrum peak frequency (e.g., Stäli et al. 1989).

In our analysis we used Ramaty's code (Ramaty 1969) to search for the magnetic field that matches the observed peak. However, we have also developed a simple expression to determine this magnetic field from the spectral peak frequency as given by the spectrum least square fit instead of opacity equal to one. This expression was only necessary to make our analysis more recurrent because the observed spectra generally present optically thick spectral indices below those predicted by the non-thermal emission theory (e.g., Dulk \& Marsh 1982) and/or some unmatched spectral broadening. Both spectral characteristics are due to a non-homogeneous magnetic field (e.g., Klein et al. 1986). Our equation differs slightly from the opacity equal to one approximation given by Dulk \& Marsh (1982). To calculate the emission and self-absorption gyro-synchrotron coefficients we used Ramaty's code and fit the calculated flux densities by the four parameter function given by Stäli et al. (1989). The flux densities were calculated supposing a homogeneous cylindrical source at one astronomical unit distance with source size $(\Omega)$ ranging from 10 to 20 arcsec wide (although the peak frequency does not depend on $\Omega$ ). The non-thermal electron energy spectrum is supposed to be a single power law with a spectral index $\delta$ in the energy $(\epsilon)$ range of $10 \mathrm{keV} \leq \epsilon \leq 10 \mathrm{MeV}$. The total flux density (the sum of both propagation modes) spectrum is calculated using Ramaty's code for five free parameters: the magnetic field ( $B_{2}$ in 100 Gauss), the source size in the line of sight ( $L_{9}$ in billions of $\mathrm{cm}$ ), the viewing angle $(\theta)$, the spectral index $(\delta)$ of the electron energy distribution and the non-thermal electron number density $\left(n_{6}\right.$ in millions of $\left.\mathrm{cm}^{-3}\right)$.

$v_{\text {peak }}=\left(n_{6} L_{9}\right)^{0.12}\left[B_{2}\left(13.5 \sin (\theta) \mathrm{e}^{-\delta}+0.9\right)+0.5 \sin (\theta)\right]$.

The errors involved in our Eq. (1) are in general below $20 \%$ and is discussed in Costa \& Rosal (2004).

\section{Viewing angle}

One important parameter for the gyro-synchrotron emission is the viewing angle of the magnetic field lines. It has been shown by the EUV instruments on board the TRACE and SOHO satellites that the field lines in active regions can vary from a simple loop to a complex tunnel of magnetic arcs. To overcome the difficulties in determining the viewing angle, we searched for the geometric conditions to minimize the viewing angle dependence. Let us assume a simple magnetic loop as shown in Fig. 4. For a simple magnetic loop, the legs can be assumed as vertical bars and the loop-top as a horizontal bar which will be called a square loop. This simplification only assumes that the bars are averaged directions of the field lines associated with each region: the loop legs and the loop-top. In our schematic figure, the $\phi$ angle is an unknown that introduces a complication for all the flares in our sample. The problem becomes worse if the flare involves a complex system of loops with unknown directions. The averaged direction of the loop-top or 


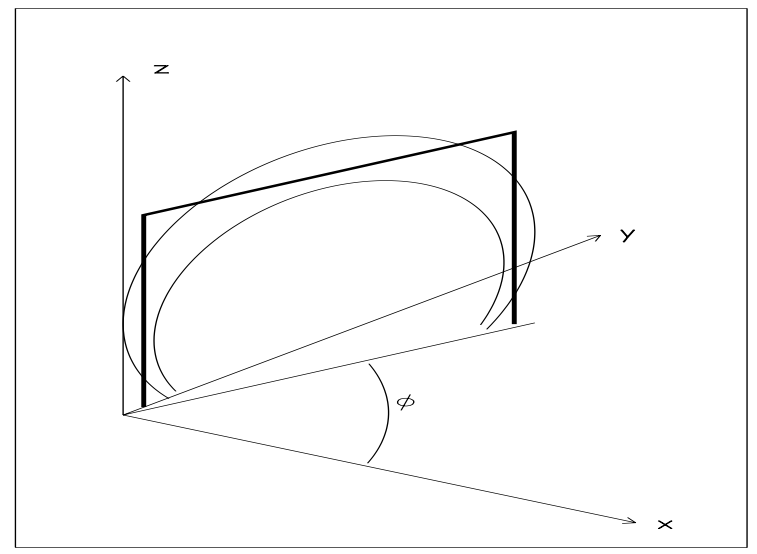

Fig. 4. Model of magnetic loop. The thick lines represent the averaged direction of the field lines in the loop legs and the loop top. The $X$-direction is aligned with the solar equator and the $Y$-direction points to the north. The observer is in $Z$-direction. The angle $\phi$ measures the alignment of the loop with the solar equator.

loop-legs is unlikely to result in a valid measurement. The viewing angle for the legs of a square loop at a heliographic latitude (lat) and longitude (long) with a loop alignment angle with respect to the equator, $\phi$, (see Fig. 4) can be found from spherical trigonometry as

$\theta_{\text {legs }}=\cos ^{-1}[\cos ($ long $) \cos ($ lat $)]$

and for the loop top as

$\theta_{\mathrm{top}}=\cos ^{-1}\left[\cos \left(\right.\right.$ long $\left.^{\prime}\right) \cos \left(\right.$ lat $\left.\left.^{\prime}\right)\right]$

where

$$
\begin{aligned}
\text { long }^{\prime} & =\text { long }+ \text { long }^{\prime \prime}-\text { long }^{\prime \prime \prime} \\
\text { long }^{\prime \prime} & =\cos ^{-1}\left[-\frac{\sin (\text { lat })}{\sin (\phi) \cos \left(\text { lat }^{\prime}\right)}\right] \\
\text { long }^{\prime \prime \prime} & =\sin ^{-1}\left[-\frac{\tan (\text { lat })}{\tan (\phi)}\right] \\
\text { lat }^{\prime} & =\sin ^{-1}\left[\sin (\phi) \sqrt{1-\left(\frac{\sin (\text { lat })}{\sin (\phi)}\right)^{2}}\right] .
\end{aligned}
$$

From Eqs. (2) and (3) we can derive the uncertainties in the viewing angle (for the top and legs) when varying the loop alignment to the equator $(\phi)$ by $90^{\circ}$. It is easy to see that a square loop at the Sun center has a viewing angle of $90^{\circ}$ for the top and $0^{\circ}$ for the legs, independently of $\phi$. Thus, we searched for the range of solar longitudes which kept the error in the inferred magnetic field below $20 \%$. This range was found to be $-30^{0} \leq$ long $\leq 30^{\circ}$. Thus, we can say that for this range of heliographic coordinates even a complex distribution of simple magnetic loops cannot influence much the viewing angle of loop legs or loop tops. We set an averaged $\phi$ angle inferred from the observed elongation of the $17 \mathrm{GHz}$ and $34 \mathrm{GHz}$ sources in NRO synthesized maps for the whole sample of 40 bursts. However, 13 events are in the range of central longitudes and were selected to have their spectra analyzed first.

\section{Spectral analysis}

The 13 events selected by the lower than $30^{\circ}$ longitude were filtered by the MRM to separate the low and fast component. Then, their spectra were fit with the four parameter function (Stäli et al. 1989), similar to the example shown in Fig. 3. To decrease the error in the spectra-fit, mainly due to errors in the fast component that is always very faint, we averaged the flux densities by seven samples $(0.7 \mathrm{~s})$. The flux densities at $80 \mathrm{GHz}$, when available, were averaged up to $\tau / 2$.

The fits yield the peak frequency $\left(v_{\mathrm{p}}\right)$ and the electron spectral index $(\delta)$ inferred from the optically thin spectral index, $\alpha_{\text {thin }}$, (e.g., Dulk \& Marsh 1982). For the optically thin part of the microwave spectrum we estimate the uncertainty $\left(\sigma_{\alpha_{-} \text {thin }}\right)$ in the inferred spectral index by the use of the general theory of error propagation applied to the power law least squares fit (Press et al. 1992). Due to the small number of frequencies in the optically thin region, we included the spectral peak frequency in the calculation of the $\sigma_{\alpha}$ thin but with a large uncertainty corresponding to the difference between the fitted power law and the measured flux. However, we emphasize that the large errors involved at $80 \mathrm{GHz}$ due to a low signal to noise ratio and background uncertainties imply that the optically thin spectral index should be analyzed with care. The $80 \mathrm{GHz}$ data does not much change the spectral peak because in all fits we had the peak below $35 \mathrm{GHz}$.

The viewing angle was calculated using Eqs. (2) and (3) with a $\phi$ angle inferred as discussed in the previous section, but it cannot influence the magnetic field much for bursts near the disk center. For the source area $(\Omega)$ and depth within the line of sight (L), we assume typical numbers such as $10^{18} \mathrm{~cm}^{2}$ and $10^{9} \mathrm{~cm}$, respectively. We emphasize that $\Omega$ does not influence the gyro-synchrotron peak frequency. For the number density of the non-thermal electrons $(n)$ we assume a common electron population emitting microwave and hard X-rays in a first order approximation to determine $n$ from HXT data (from YOHKOH satellite). We applied the thick target model (Brown 1971) to hard X-ray spectra for 7 events from our sample of 13. Following the formalism described in Tandberg-Hanssen \& Emslie (1988), we assume a photon power law spectrum with a lower energy cutoff of $20 \mathrm{keV}$ and a hard X-ray source size of $10^{8} \mathrm{~cm}$. The electron number densities were calculated for the flare peaks using the HXT energy bands $M_{1}: 23-33 \mathrm{keV}$ and $M_{2}: 33-53 \mathrm{keV}$ and are presented in Table 2. For the other 6 events the density was assumed to be the mean value of the bursts in Table 2, i.e., $n_{6}=60 \mathrm{~cm}^{-3}$.

\section{Results and discussion}

The inferred spectral index and magnetic field were obtained; the results are shown in Table 3. The table contains the average of the parameters for the three main pulse structures in each flare weighted by the inverse of the standard deviation of each parameter. In this table we can observe that the spectral index $\delta$ is higher for the fast component (loop-leg) in 9 out of 13 cases, mean values being $\delta_{\text {top }}=3.3 \pm 0.1$ for the loop-top and $\delta_{\text {foot }}=4.1 \pm 0.1$ for the loop-legs. The magnetic fields are also higher in the loop-legs, as expected, with mean values of 
Table 2. Inferred electron number density assuming a bremstrhalung thick target model. The $n_{6}$ is the number density given in units of $10^{6} \mathrm{~cm}^{-3}$.

\begin{tabular}{cccccccc}
\hline \hline$\#$ & 11 & 15 & 26 & 28 & 32 & 35 & 39 \\
\hline$\delta$ & 6.4 & 4.7 & 4.7 & 4.9 & 4.3 & 4.2 & 4.7 \\
$n_{6}$ & 2 & 60 & 50 & 50 & 200 & 50 & 8 \\
\hline
\end{tabular}

Table 3. Inferred spectra parameters for thirteen events located at solar longitudes from $30^{\circ}$ East to $30^{\circ}$ West.

\begin{tabular}{c|ccc|cccc}
\hline \hline & \multicolumn{3}{|c|}{ From top } & \multicolumn{4}{c}{ From legs } \\
$\#$ & $\delta$ & $v_{\mathrm{p}}$ & $B_{2}$ & $\delta$ & $v_{\mathrm{p}}$ & $B_{2}$ & M.R. \\
\hline 7 & 2.9 & 10.0 & 3.2 & 3.8 & 12.7 & 8.7 & 2.7 \\
12 & 3.6 & 12.3 & 12.9 & 2.5 & 21.6 & 15.3 & 1.2 \\
13 & 3.2 & 5.3 & 1.8 & 3.8 & 9.1 & 5.8 & 3.3 \\
16 & 3.7 & 11.0 & 5.0 & 4.8 & 18.8 & 12.4 & 2.5 \\
17 & 3.8 & 8.3 & 4.4 & 3.8 & 10.2 & 6.5 & 1.5 \\
27 & 3.3 & 15.1 & 5.3 & 3.8 & 16.0 & 11.8 & 3.0 \\
30 & 2.8 & 12.6 & 4.3 & 4.2 & 18.3 & 13.4 & 3.1 \\
32 & 3.7 & 9.1 & 4.7 & 3.6 & 10.7 & 6.6 & 1.4 \\
33 & 4.0 & 10.4 & 5.8 & 3.0 & 12.1 & 7.6 & 1.4 \\
34 & 3.9 & 12.2 & 5.1 & 4.2 & 15.8 & 9.2 & 1.8 \\
36 & 2.8 & 6.0 & 1.7 & 3.7 & 8.7 & 6.0 & 3.5 \\
39 & 4.3 & 11.3 & 10.6 & 4.0 & 12.0 & 13.1 & 1.2 \\
40 & 4.4 & 21.4 & 14.1 & 5.6 & 27.4 & 21.6 & 1.5 \\
\hline
\end{tabular}

$B_{\text {top }}=600$ Gauss for the loop-top and $B_{\text {foot }}=1060$ Gauss for the loop-legs, giving a mirror ratio of 1.8. The harder spectra (on average) at the loop-top favors the hypothesis of Coulomb diffusion of the electron pitch angle in the trap. Electrons with higher energies are trapped for longer periods than the slow electrons and consequently are depleted from the loop-legs (e.g., Lee \& Gary 2000). This result is not in disagreement with Silva et al. (2000) measurements from X rays and microwave spectra. They found that, in general, the electronic spectrum is harder for the high energy range (as inferred from microwaves) than for the lower energy range (as inferred from hard $\mathrm{X}$ rays). Here, we found a spatial hardening in the direction of the loop top from the hypothesis of a single power law that also should work for any other shape of spectrum. The magnetic fields for the loop-top are typical of most of the analysis in the literature with the exception of bursts number 11 and 40. The number density for burst 40 was not inferred from X-rays and may be underestimated. This burst is the second most intense burst in our sample. However, burst number 11 has an electronic spectral index inferred from $X$ rays much higher than the same from microwaves and we consider it not appropriate for the analysis. For the loop-legs the magnetic fields are higher but consistent with the expectation. The magnetic field intensity should not be considered as too high because this is the first time that we consider the spectrum of the fast structures only. The fast structures are only a small part of the total flux density and cannot modify the bulk spectra. Kai et al. (1985) correlate the fast structures at $17 \mathrm{GHz}$ with X-rays and concluded that the magnetic field must be higher than the typical values used in the literature. Also, the mirror ratio found here is similar to other analyses found in the literature (e.g., Aschwanden et al. 1999). For the whole sample of bursts, assuming the main orientation of the loop with a defined alignment according to our inference from the image elongation in the synthesized NRO maps, $\delta_{\text {top }}=3.2 \pm 0.1$, $\delta_{\text {foot }}=4.0 \pm 0.1, B_{\text {top }}=580$ Gauss and $B_{\text {foot }}=940$ Gauss. These figures confirm the results from the central bursts.

Acknowledgements. We are grateful to the NoRP and YOHKOH staff for the solar flare measurements with the Nobeyama Radio Polarimeter and HXT of the YOHKOH satellite and making them available over the Internet. The Nobeyama Radio Polarimeter is operated by NAOJ/Nobeyama Solar Radio Observatory. We thank the anonymous referee for the great improvement of the text.

\section{References}

Aschwanden, M. J. 1998, ApJ, 502, 455

Aschwanden, M. J., Schwartz, R. A., \& Alt, D. M. 1995, ApJ, 447, 923

Aschwanden, M. J., Fletcher, L., Sakao, T., Kosugi, T., \& Hudson, H. 1999, ApJ, 517, 977

Bai, T., \& Ramaty, R. 1979, ApJ, 227, 1072

Brown, J. C. 1971, Sol. Phys., 18, 489

Brown, J. C. 1973, Sol. Phys., 28, 151

Correia, E., \& Kaufmann, P. 1987, Sol. Phys., 111, 143

Costa, J. E. R., \& Rosal, A. C. 2004, in the proceedings of the Magnetic Fields in the Universe Workshop held in Angra do Reis, Brazil, 28 November to 3 December, 2004, in press

Costa, J. E. R., Brown, J. C., Correia, E., \& Kaufmann, P. 1990, ApJS, 73,191

Datlowe, D. W., \& Lin, R. P. 1973, Sol. Phys., 32, 459

Dulk, G. A., \& Marsh, K. A. 1982, ApJ, 23, 169

Gary, D. 1985, ApJ, 297, 799

Hoyng, P., Van Beek, H. F., \& Brown, J. C. 1976, Sol. Phys., 48, 197

Kai, K., Kosugi, T., \& Nitta, N. 1985, PASJ, 37, 155

Kaufmann, P. 1978, Sol. Phys., 60, 367

Kaufmann, P., Costa, J. E. R., \& Strauss, F. M. 1982, Sol. Phys., 81, 159

Kaufmann, P., Costa, J. E. R., Dennis, B. R., et al. 1983, Sol. Phys., 84,311

Klein, K.-L., Trottet, G., \& Magun, A. 1986, Sol. Phys., 104, 243

Kiplinger, A. L., Dennis, B. R., Emslie, A. G., Frost, K. J., \& Orwig, L. E. 1983, ApJ, 265, L99

Kundu, M. R. 1961, J. Geophys. Res., 66, 4308

Leach, J., \& Petrosian, V. 1981, ApJ, 251, 781

Leach, J., \& Petrosian, V. 1983, ApJ, 269, 715

Lee, J., \& Gary, D. E. 2000, ApJ, 543, 457

Lin, R. P., \& Johns, C. M. 1993, ApJ, 327, 405

Lu, E. T., \& Petrosian, V. 1988, ApJ, 327, 405

MacKinnon, A. L., Brown, J. C., Trottet, G., \& Vilmer, N. 1983, A\&A, 119, 297

Melrose, D. B., \& Brown, J. C. 1976, MNRAS, 176, 15

Nakajima, H., Sekiguchi, H., Sawa, M., Kai, K., \& Kawashima, S. 1985, PASJ, 37, 163

Press, W. H., Teukolsky, S. A., Vetterling, W. T., \& Flannery, B. P. 1992, Numerical Recipes (Cambridge: University Press)

Ramaty, R. 1969, ApJ, 158, 753

Raulin, J. P., Wilson, R. F., Kerdraon, A., et al. 1991, A\&A, 251, 298

Stähli, M., Gary, D. E., \& Hurford, G. J. 1989, Sol. Phys., 120, 351

Silva, A. V. R., Wang, H., \& Gary, D. E. 2000, ApJ, 545, 1116

Tandberg-Hanssen, E., \& Emslie, A. G. 1988, The Physics of Solar Flares (Cambridge: University Press)

Takakura, T., \& Kai, K. 1966, PASJ, 18, 57

Trubnikov, B. A. 1965, Rev. Plasma Phys., 1, 205 
J. E. R. Costa and A. C. Rosal: microwave trap plus precipitation burst emission, Online Material p 1

\section{Online Material}


J. E. R. Costa and A. C. Rosal: microwave trap plus precipitation burst emission, Online Material p 2

Table 1. Analyzed events. The name used is taken from the flare list on the NRO web-site: solar.nro.nao.ac.jp/norp/html/event/. The second column has the cutoff period in seconds and the third, fourth columns are the flare duration and the spectrum peak flux density, respectively.

\begin{tabular}{ccccc}
\hline \hline$\#$ & Event & $\tau$ & $\begin{array}{c}\text { Duration } \\
(\mathrm{s})\end{array}$ & $\begin{array}{c}\text { Intensity } \\
(\mathrm{sfu})\end{array}$ \\
\hline 1 & norp19980808_0315 & 1.7 & 89 & 1997 \\
2 & norp19980909_0456 & 2.1 & 238 & 303 \\
3 & norp19981112_0527 & 0.8 & 36 & 248 \\
4 & norp19981122_0638 & 2.2 & 392 & 13526 \\
5 & norp19981124_0214 & 2.5 & 545 & 1784 \\
6 & norp19981128_0539 & 1.8 & 954 & 3403 \\
7 & norp19990216_0001 & 1.9 & 241 & 245 \\
8 & norp19990311_0008 & 2.7 & 44 & 43 \\
9 & norp19990404_0521 & 3.0 & 326 & 344 \\
10 & norp19990517_0453 & 2.5 & 173 & 593 \\
11 & norp19990626_0511 & 1.1 & 50 & 47 \\
12 & norp19990702_0133 & 3.4 & 332 & 197 \\
13 & norp19990728_0156 & 3.5 & 933 & 305 \\
14 & norp19990804_0549 & 2.0 & 226 & 577 \\
15 & norp19990820_2306 & 0.5 & 55 & 56 \\
16 & norp19990825_0135 & 0.7 & 55 & 132 \\
17 & norp19990828_0056 & 1.3 & 593 & 1195 \\
18 & norp19990917_0118 & 2.0 & 318 & 198 \\
19 & norp19991025_0629 & 1.4 & 250 & 145 \\
20 & norp19991116_0408 & 2.5 & 78 & 244 \\
21 & norp19991122_0254 & 1.0 & 38 & 179 \\
22 & norp19991227_0150 & 2.0 & 284 & 578 \\
23 & norp19991228_0043 & 1.2 & 154 & 6623 \\
24 & norp20000112_0136 & 2.5 & 236 & 911 \\
25 & norp20000120_0224 & 1.0 & 33 & 268 \\
26 & norp20000303_0212 & 2.0 & 232 & 1448 \\
27 & norp20000313_0503 & 1.5 & 65 & 26 \\
28 & norp20000315_0540 & 1.3 & 14 & 19 \\
29 & norp20000323_0028 & 3.8 & 48 & 88 \\
30 & norp20000408_0238 & 2.9 & 313 & 688 \\
31 & norp20000618_0156 & 1.2 & 143 & 754 \\
32 & norp20000623_0404 & 1.5 & 293 & 536 \\
33 & norp20000721_0522 & 1.2 & 67 & 36 \\
34 & norp20000725_0247 & 1.4 & 195 & 3019 \\
35 & norp20000727_0408 & 1.5 & 82 & 576 \\
36 & norp20000916_0415 & 2.8 & 1022 & 2778 \\
37 & norp20000930_2319 & 2.8 & 193 & 5217 \\
38 & norp20001029_0147 & 2.8 & 1302 & 1683 \\
39 & norp20001123_2321 & 2.4 & 154 & 697 \\
40 & norp20001124_0459 & 2.3 & 135 & 8638 \\
\hline & & &
\end{tabular}

